\title{
Correction
}

\section{Correction: Seger et al., Generalization in Category Learning: The Roles of Representational and Decisional Uncertainty}

In the article "Generalization in Category Learning: The Roles of Representational and Decisional Uncertainty" by Carol A. Seger, Kurt Braunlich, Hillary S. Wehe, and Zhiya Liu, which appeared on pages 8802-8812 of the June 10, 2015 issue, the authors regret that Dr. Kurt Braunlich's, Dr. Hillary S. Wehe's, and Dr. Zhiya Liu's affiliations were switched. Their corrected affiliations are as follows: Dr. Kurt Braunlich and Dr. Hillary S. Wehe, Department of Psychology and Program in Molecular, Cellular, and Integrative Neurosciences, Colorado State University, Fort Collins, Colorado 80523; and Dr. Zhiya Liu, Center for the Study of Applied Psychology, Key Laboratory of Mental Health and Cognitive Science of Guangdong Province, School of Psychology, South China Normal University, Guangzhou 510631, P.R. China.

DOI:10.1523/JNEUROSCI.2729-15.2015 